\title{
Depot Buprenorphine Injection in the Management of Opioid Use Disorder: From Development to Implementation [Corrigendum]
}

Ling W, Shoptaw S, Goodman-Meza D. Subst Abuse Rehabil. 2019;10:69-78.

The authors of this paper have advised that Table 1 is incorrect. The authors advised that they had listed values for Cmax and Ctrough after a single dose of Brixadi
(CAM2038) and not steady state, as it was provided for Sublocade. The new Table 1 reflects the Brixadi steady state concentrations for a more direct comparison with Sublocade.

The correct Table 1 is as follows:

Table I Comparison of Long-Acting Formulations of Buprenorphine FDA-Approved for Treatment of Opioid Use Disorder

\begin{tabular}{|c|c|c|c|}
\hline Brand Name & Probuphine & Sublocade & Brixadi (US) or Buvidal (Europe/Australia) \\
\hline Molecular name & & RBP-6000 & CAM2038 \\
\hline Pharmaceutical & Previously Braeburn, currently Titan & Indivior & Braeburn Pharmaceuticals/Camurus \\
\hline $\begin{array}{l}\text { Indicated } \\
\text { population }\end{array}$ & $\begin{array}{l}\text { Stable transmucosal buprenorphine } \\
\text { dose of } 8 \mathrm{mg} \text { or less for three } \\
\text { months or longer }\end{array}$ & $\begin{array}{l}\text { Initiated transmucosal } \\
\text { buprenorphine }(8-24 \mathrm{mg}) \text { for } \\
\text { a minimum of } 7 \text { days. }\end{array}$ & $\begin{array}{l}\text { Initiation of treatment in patients not already } \\
\text { receiving buprenorphine or switching from } \\
\text { transmucosal buprenorphine }\end{array}$ \\
\hline $\begin{array}{l}\text { Route of } \\
\text { administration }\end{array}$ & Subcutaneous implant & Subcutaenous injection & Subcutaenous injection \\
\hline Duration of effect & 6 months & I month & I week or I month \\
\hline Dosage & $320 \mathrm{mg}$ (Four $80 \mathrm{mg}$ implants) & 100 and $300 \mathrm{mg}$ & $\begin{array}{l}\text { 8, 16, } 24 \text { and } 32 \mathrm{mg} \text { (weekly) or 64, } 96 \text { and } 128 \mathrm{mg} \\
\text { (monthly) }\end{array}$ \\
\hline $\begin{array}{l}\text { Long acting } \\
\text { technology }\end{array}$ & $\begin{array}{l}\text { Ethylene vinyl acetate (EVA) } \\
\text { polymer }\end{array}$ & $\begin{array}{l}\text { I8\% (weight/weight) } \\
\text { buprenorphine base in the } \\
\text { ATRIGEL Delivery System }\end{array}$ & $\begin{array}{l}\text { Prolonged release FluidCrystal injection depot } \\
\text { technology }\end{array}$ \\
\hline Location & Upper arm & Abdomen & Buttock, thigh, stomach (abdomen) or upper arm \\
\hline FDA-approval & 2016 & 2017 & 2018 (tentative) \\
\hline $\begin{array}{l}\text { Plasma } \\
\text { concentrations } \\
(\mathrm{ng} / \mathrm{mL})\end{array}$ & $\begin{array}{l}\mathrm{C}_{\max } \\
3.23 \\
\mathrm{C}_{\text {trough }} \\
0.72\end{array}$ & $\begin{array}{l}C_{\max } \\
4.88(100 \mathrm{mg}) \\
10.12(300 \mathrm{mg}) \\
C_{\text {trough }} \\
2.48(100 \mathrm{mg}) \\
5.01(300 \mathrm{mg})\end{array}$ & $\begin{array}{l}C_{\max } \\
\text { Weekly } 4.3-6.9 \\
\text { Monthly } 4.0-11.1 \\
C_{\text {trough }} \\
\text { Weekly } 0.8-2.6 \\
\text { Monthly } 1.3-2.1\end{array}$ \\
\hline
\end{tabular}

(Continued) 
Table I (Continued).

\begin{tabular}{|c|c|c|c|}
\hline Brand Name & Probuphine & Sublocade & Brixadi (US) or Buvidal (Europe/Australia) \\
\hline Provider burden & $\begin{array}{l}+++ \\
\text { Live training program } \\
\text { Procedural competency }\end{array}$ & $\begin{array}{l}++ \\
\text { Supervised injection } \\
\text { Monthly injections }\end{array}$ & $\begin{array}{l}++ \\
\text { Supervised injection } \\
\text { Weekly or monthly injections }\end{array}$ \\
\hline $\begin{array}{l}\text { Special Handling } \\
\text { Requirements }\end{array}$ & $\begin{array}{l}\text { Requires implant procedure } \\
\text { Need for removal or replacement } \\
\text { every } 6 \text { months }\end{array}$ & $\begin{array}{l}\text { Needs Refrigeration } \\
\text { Injection only under skin } \\
\text { around umbilicus }\end{array}$ & No special requirements \\
\hline
\end{tabular}

\section{Publish your work in this journal}

Substance Abuse and Rehabilitation is an international, peerreviewed, open access journal publishing original research, case reports, editorials, reviews and commentaries on all areas of addiction and substance abuse and options for treatment and rehabilitation. The manuscript management system is completely online and includes a very quick and fair peer-review system. Visit http://www.dovepress com/testimonials.php to read real quotes from published authors. 